\title{
ADUBAÇÃO DE CRESCIMENTO DE MACIEIRA CV. CATARINA SOBRE PORTA-ENXERTO MARUBAKAIDO EM SÃO JOAQUIM-SC ${ }^{1}$
}

\author{
GILBERTO NAVA ${ }^{2}$, NÉVIO JOÃO NUERNBERG ${ }^{3}$, ADILSON JOSÉ PEREIRA $^{4}$, ANTONIO ROQUE DECHEN ${ }^{5}$
}

\begin{abstract}
RESUMO - O vigor vegetativo e a capacidade produtiva da macieira são dependentes da adubação de crescimento ou de formação, a qual se baseia no fornecimento de N. A macieira cultivar Catarina, selecionada como resistente à sarna, tem-se mostrado promissora para as regiões de maior altitude do Sul do Brasil, mas, por tratar-se de uma cultivar nova, ainda não existem recomendações de adubação para a formação inicial das plantas. Por essa razão, conduziu-se um experimento com o objetivo de estabelecer a dose de $\mathrm{N}$ capaz de equilibrar o vigor vegetativo com a capacidade produtiva da planta. O experimento foi instalado num pomar estabelecido de macieira cultivar Catarina, enxertada sobre o porta-enxerto Marubakaido, implantado em 1998, num solo raso originário de rochas magmáticas ácidas, no município de São Joaquim. O solo (Neossolo) havia sido corrigido quanto ao pH e teores de fósforo e de potássio, de acordo com as recomendações para macieira. Avaliaram-se as doses de N: zero; 25; 50 e $100 \mathrm{~kg}^{-1}$ (soma de três aplicações anuais a partir do ano subseqüente ao plantio, na projeção da copa das plantas). As parcelas foram formadas por oito plantas em blocos ao acaso e oito repetições. O crescimento dos ramos do ano das plantas, o número de esporões e brindilas não foram afetados pelas doses de N. Todavia, conjeturou-se que a adubação nitrogenada, nos anos anteriores, afetou a nutrição das gemas florais, dado o aumento significativo no número de frutos no primeiro ciclo de produção das plantas.
\end{abstract}

Termos para indexação: N, macieira, adubação de crescimento.

\section{GROWING FERTILIZATION FOR “CATARINA” APPLE TREES GRAFTED ONTO MARUBAKAIDO ROOTSTOCK IN SÃO JOAQUIM, SC, BRAZIL}

\begin{abstract}
The vegetative vigor and the yield potential of the apple trees primarily depend on the initial soil fertilization (growing or plant formation fertilization), which is based mainly on nitrogen application. The "Catarina" apple cultivar selected as scab resistant, seems to be a promising cultivar for higher altitude areas in Southern Brazil. As a new cultivar, fertilizer recommendations for the initial plant formation have not been available yet. For this reason, an experiment was carried out to establish the nitrogen requirement to balance both vegetative vigor and the plant yield potential. The orchard was planted in August 1998 in a shallow soil originated from acid magmatic rocks, in São Joaquim - SC, Brazil. The cultivar studied was Catarina grafted onto Marubakaido rootstock. The area had been limed to elevate the soil $\mathrm{pH}$; phosphorous and potassium were supplied according to fertilizer recommendations. The $\mathrm{N}$ rates tested were: $0,25,50$ and $100 \mathrm{~kg} \mathrm{ha}^{-1}$ (sum of three annual applications beginning from the subsequent year to the plantation), placed on the projection of the tree canopy. Eight plants organized in randomized blocks design with eight replications formed the plots. The branches growth of the year and the number of spurs were not affected by any $\mathrm{N}$ rates. However, it was supposed that the nitrogen fertilization from previous years had affected the fruit buds nutrition, considering the significant increasing of the number of fruits and the yield of the trees on first year of production.
\end{abstract}

Index terms: nitrogen, apple trees, young tree fertilization.

\section{INTRODUÇÃO}

A adubação de crescimento em macieiras tem por objetivo propiciar as melhores condições nutricionais para uma boa formação da planta durante a fase que antecede o início da produção, a qual dará suporte às produções futuras. Pomares implantados em solos corretamente corrigidos na fase de implantação com calcário e fertilizantes, basicamente, apenas necessitarão de adubações suplementares com $\mathrm{N}$ durante o período que antecede o início da produção (Suzuki \& Basso, 2002). Durante a fase de formação das plantas, tanto o excesso quanto a falta de $\mathrm{N}$ pode refletir em decréscimos na produtividade e na qualidade das maçãs nos anos subseqüentes. O teor excessivo de $\mathrm{N}$ em plantas jovens retarda a entrada em produção e deprime a formação de estruturas de órgãos reprodutivos, diminuindo, conseqüentemente, o número de frutos por planta, os quais tendem a ser maiores, porém mais suscetíveis à ocorrência de desordens fisiológicas (Iuchi et al., 2001). O excesso de $\mathrm{N}$ estimula demasiadamente o crescimento de órgãos vegetativos (excesso de folhagem e ramos verticais conhecidos como ladrões). Um adequado nível de $\mathrm{N}$ é fundamental para a diferenciação de gemas (Manru et al., 1990) e crescimento das plantas (Stiles, 1994; Daugaard \& Grauslund, 2000). Plantas com deficiência de N

\footnotetext{
${ }^{1}$ (Trabalho 088-06). Recebido em : 28-06-2006. Aceito para publicação em : 05-04-2007.

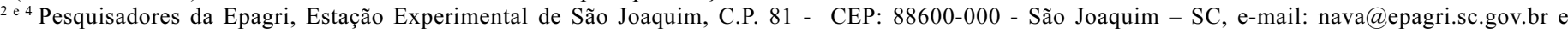
Pereira@epagri.rct-sc.br

${ }^{4}$ Pesquisador da Epagri, C. P. 502 - CEP: 88034-901- Florianópolis - SC, e-mail: nevio@epagri.rct-sc.br

${ }^{5}$ Professor USP/ESALQ - Depto. de Solos e Nutrição de Plantas, C.P. 9 - CEP: 13418-900 - Piracicaba,, SP, e-mail:ardechen@esalq.usp.br
}

Rev. Bras. Frutic., Jaboticabal - SP, v. 29, n. 2, p. 359-363, Agosto 2007 
demoraram mais para entrar em frutificação que plantas com teores normais de N (Manru et al., 1990).

No Brasil, inexistem trabalhos procurando avaliar a necessidade de adubação de crescimento para a macieira. Os trabalhos feitos até então concentram-se na avaliação da necessidade de adubações em manutenção. Para esses casos, a resposta em termos de rendimento em função da adubação nitrogenada tem sido não-significativa em solos profundos das regiões de Fraiburgo e Vacaria (Basso \& Suzuki, 1992; Ernani et al., 1997; Proença et al., 2002). Para as condições de solos pouco profundos da região de São Joaquim-SC, a adubação nitrogenada em manutenção tem sido favorável ao rendimento da macieira (Nava et al., 2000).

É importante ressaltar o fato de que plantas jovens possuem um sistema radicular menos desenvolvido do que plantas adultas e, conseqüentemente, exploram menor volume de solo. A contribuição do $\mathrm{N}$ armazenado em órgãos de reserva no ciclo anterior e que é remobilizado para os tecidos na fase inicial da estação de crescimento, é baixo para plantas jovens (Neilsen et al., 2001). Esses fatores podem aumentar a exigência de $\mathrm{N}$ suplementar durante os primeiros anos após o plantio das plantas. Além disso, as menores temperaturas médias observadas durante o ano, na região de São Joaquim, em relação às demais regiões produtoras de maçã do Brasil, refletem em menor taxa de mineralização da matéria orgânica do solo.

As recomendações atuais de adubação nitrogenada em crescimento para a macieira, no Brasil sugerem quantidades de $18 ; 27$ e $36 \mathrm{~kg} \mathrm{ha}^{-1} \mathrm{ano}^{-1}$, para o $1^{\circ}, 2^{\circ}$ e $3^{\circ}$ anos após o plantio das mudas, respectivamente (Comissão de Química e Fertilidade do Solo, 2004). A cultivar Catarina tem o hábito de crescimento vegetativo mais vigoroso que as cultivares Gala e Fuji, tradicionalmente os mais cultivados no Brasil. Por isso, a exigência de $\mathrm{N}$ da cultivar Catarina pode ser menor.

Devido à inexistência de parâmetros técnicos para recomendação de adubação de crescimento, realizou-se o presente experimento com o objetivo de avaliar a necessidade de $\mathrm{N}$ para a formação inicial das plantas e sua influência sobre o primeiro ano de produção da macieira, cv. Catarina, para as condições do Planalto Serrano de São Joaquim.

\section{MATERIAL E MÉTODOS}

O experimento foi instalado num pomar de macieira cultivar Catarina, enxertada sobre o porta-enxerto Marubakaido, implantado em 1998, num solo raso originário de rochas magmáticas ácidas (Neossolo), no município de São Joaquim - SC (28 17' 25" S, 49 56' 56" W), tendo o $\mathrm{pH}$ corrigido (30 $\left.\mathrm{Mg} \mathrm{ha}^{-1}\right)$, o P e o K com 325 e $200 \mathrm{~kg} \mathrm{ha}^{-1}$ de $_{2} \mathrm{O}_{5}$ e $\mathrm{K}_{2} \mathrm{O}$, respectivamente, e boro com $30 \mathrm{~kg} \mathrm{ha}^{-1}$ de bórax. Nos três anos subseqüentes ao do plantio, utilizaram-se doses crescentes de $\mathrm{N}$, as quais totalizaram no período: zero; 25; 50 e $100 \mathrm{~kg} \mathrm{ha}^{-1}$ (soma das aplicações dos três anos), aplicadas superficialmente ao solo, na projeção da copa das plantas, e parceladas em três aplicações anuais, nas épocas recomendadas pela Comissão de Fertilidade do Solo (1995). As parcelas formadas por oito plantas foram arranjadas num delineamento experimental completamente casualizado, com oito repetições. As plantas foram manejadas pelo produtor de acordo com seu interesse, exceto em relação à adubação nitrogenada.

Nos dois anos subseqüentes ao início dos tratamentos, avaliou-se o crescimento das plantas por meio da medição da expansão do diâmetro dos troncos das árvores, a quinze centímetros acima do ponto de enxertia, do crescimento dos lançamentos do ano e da medida transversal e longitudinal da copa, que foram posteriormente utilizadas na estimativa da capacidade produtiva (CP). A CP foi obtida pelo produto da altura média da copa e do diâmetro médio da planta $(\mathrm{CP} /$ planta $=$ diâmetro médio da copa $\mathrm{x}$ altura produtiva média da copa) (Ebert \& Raasch, 1987). Também, avaliou-se o número de esporões e de brindilas em dois ramos pré-identificados de cada planta e num total de doze por parcela. No terceiro ano após o plantio (fase inicial de produção), o número de frutos por planta foi determinado na semana que antecedeu a colheita (todos os frutos de todas as plantas da parcela foram contados). Para a estimativa da massa média dos frutos, 100 frutos foram amostrados aleatoriamente. A massa e o número médio de frutos por planta foram posteriormente utilizados para a estimativa da produtividade.

No segundo e terceiro anos após o início da aplicação dos tratamentos, foram realizadas amostragens de folhas para a quantificação do teor de N. As amostras de folhas foram coletadas na parte mediana dos ramos do ano, durante o período de 15 de janeiro a 15 de fevereiro, conforme metodologia sugerida por Suzuki \& Basso (2002). Em cada parcela experimental, foram coletadas 50 folhas que foram secadas em estufa à temperatura de $65{ }^{\circ} \mathrm{C}$ até atingirem peso constante.

Os dados foram submetidos à análise da variância e regressão polinomial ao nível de significância $\alpha=0,05$, além de correlações entre variáveis de interesse, utilizando-se do programa estatístico SAS ${ }^{\circledR}$ (SAS Institute, 1996).

\section{RESULTADOS E DISCUSSÃO}

A adubação nitrogenada promoveu incremento no crescimento das plantas invasoras situadas sob as macieiras, o que tornou necessário maior número de operações de roçada na área experimental, principalmente nos tratamentos que receberam as maiores doses de $\mathrm{N}$. Uma vez que não se utilizou herbicida para controle de plantas invasoras, roçadas foram realizadas periodicamente para manter-se a altura das plantas, debaixo da projeção da copa da macieira, inferior a $10 \mathrm{~cm}$. No entanto, mesmo assim houve competição das plantas invasoras com a macieira por água e nutrientes. Isso pode ser confirmado pela análise foliar realizada no segundo ano, a qual mostrou que somente com doses superiores a $50 \mathrm{~kg} \mathrm{ha}^{-1}$ de $\mathrm{N}$, o limite inferior de $20 \mathrm{~g} \mathrm{~kg}^{-1}$ de $\mathrm{N}$ da faixa de concentração considerada normal foi atingido (SBCS: Comissão de Química e Fertilidade do Solo, 2004) (Figura 1). Para o terceiro ano, o limite inferior somente foi atingido com a dose máxima de $100 \mathrm{~kg} \mathrm{ha}^{-1}$ de $\mathrm{N}$. 


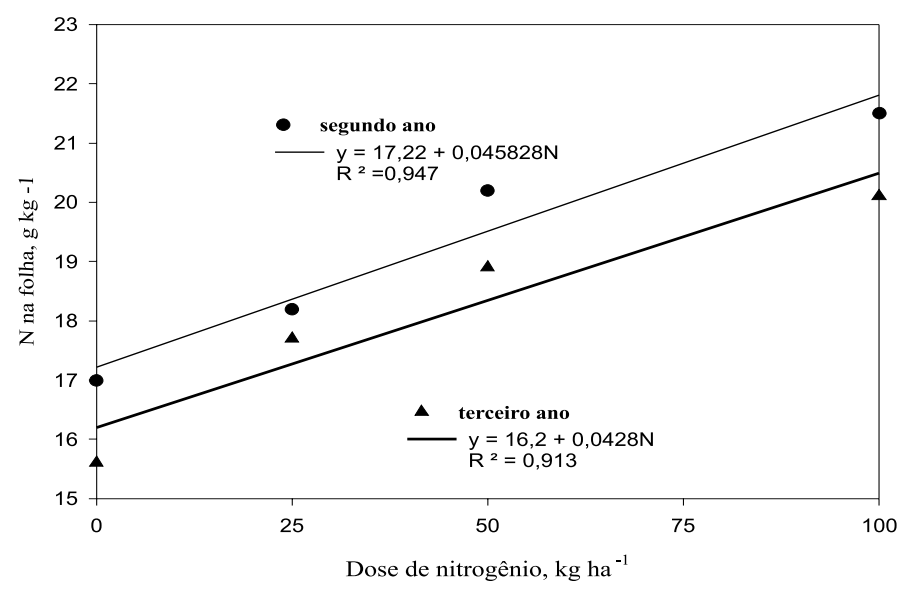

FIGURA 1 - Teores de $\mathrm{N}$ na folha de macieira em função das doses deste nutriente aplicadas ao solo durante a fase não-produtiva da cultura para as condições de São Joaquim-SC (2000 e 2001).

Sintomas visuais de deficiência de N, caracterizados pelo amarelecimento generalizado da planta foram observados nas parcelas que não receberam adubação e nas menores doses de N. Estes resultados indicam que, apesar de a cultivar Catarina ser mais vigorosa que as cultivares Gala e Fuji, ainda assim há necessidade de adubações com $\mathrm{N}$ durante a fase de estabelecimento das plantas, principalmente quando o controle de invasoras é realizado por meio de roçadas, como foi o caso do presente trabalho. No entanto, é importante ressaltar que o experimento foi realizado num solo pouco profundo, sendo que, em observações de campo com esta mesma cultivar, porém, em solos mais profundos e localizados em áreas mais planas do relevo (solos com maior teor de matéria orgânica e maior teor de umidade), tenderam a fornecer suficiente $\mathrm{N}$ durante a fase de formação das plantas.

O número de estruturas reprodutivas: esporões e brindilas não foram influenciados significativamente pela adubação nitrogenada. Em relação às variáveis de crescimento da planta, somente o perímetro do tronco aumentou em função das doses de $\mathrm{N}$ (Tabela 1). O vigor da macieira, baseado no comprimento dos lançamentos de ramos e da capacidade produtiva, foi de certa forma controlado pelo manejo das árvores e não foi afetado pela adubação nitrogenada de crescimento (Tabela 2). Ressaltase que a poda foi pouco intensa e a competição por $\mathrm{N}$ e água causada pelas plantas invasoras com a macieira, como já abordado anteriormente.

TABELA 1 - Resultado da análise da variância na resposta de diferentes atributos de planta às doses de $\mathrm{N}$ aplicadas anualmente em crescimento, para as condições de São Joaquim- SC (2000 e 2001).

\begin{tabular}{lcc}
\hline Variável medida & Doses N & CV \\
\hline & $\operatorname{Pr}>\mathrm{F}$ & \\
\hline Incremento no perímetro do tronco/segundo ano & 0,0033 & 9,72 \\
Incremento no perímetro do tronco/ terceiro ano & 0,0044 & 9,14 \\
Comprimento dos lançamentos & $\mathrm{ns}$ & 9,81 \\
Capacidade produtiva da copa & $\mathrm{ns}$ & 16,36 \\
\hline
\end{tabular}

O perímetro dos troncos das macieiras aumentou em cerca de $43 \mathrm{~mm}$ do segundo para o terceiro ano na dose zero de $\mathrm{N}$ (Figura 2). $\mathrm{O}$ incremento em função das doses de $\mathrm{N}$ foi praticamente idêntico nos dois anos, sendo 0,153 e $0,206 \mathrm{~mm}$ para cada quilograma de $\mathrm{N}$ aplicado, respectivamente, para o segundo e terceiro anos. Estes resultados corroboram aqueles apresentados por Neilsen et al. (1999), que também observaram aumento da área de secção do tronco da macieira em resposta à adubação nitrogenada. Como o vigor das plantas tende a relacionar-se positivamente com o perímetro do tronco, presumese que o vigor das plantas foi afetado pela adubação nitrogenada.

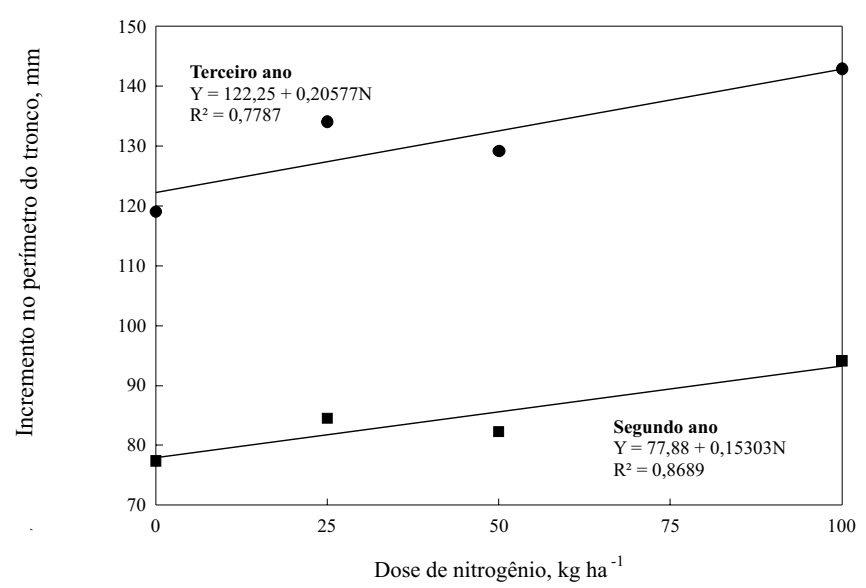

FIGURA 2 - Incremento no perímetro do tronco das macieiras cv. Catarina sobre porta-enxerto Marubakaido em função de doses de N, para as condições de São Joaquim-SC (2000 e 2001).

No entanto, o aumento no perímetro dos troncos das macieiras não refletiu no aumento do crescimento de lançamentos do ano e da capacidade produtiva da copa (CP) das árvores (Tabela 2), possivelmente devido ao manejo adotado pelo produtor, que visou a reduzir o crescimento das plantas em função do espaçamento adotado na implantação do pomar.

TABELA 2 - Valores médios das variáveis medidas que não apresentaram diferenças significativas pela análise da variância, ao nível de $5 \%$ de probabilidade, para as condições de São Joaquim-SC (2000 e 2001).

\begin{tabular}{cccc}
\hline Nitrogênio & $\begin{array}{c}\text { Crescimento dos } \\
\text { lançamentos }\end{array}$ & Esporões & $\begin{array}{c}\text { Capacidade produtiva } \\
\text { da copa }\end{array}$ \\
\hline $\mathrm{kg} \mathrm{ha}^{-1}$ & $\mathrm{Cm}$ & Número por ramo & $\mathrm{m}^{3}$ \\
0 & $38,8^{*}$ & $8,0^{*}$ & $2,5^{*}$ \\
25 & 40,8 & 7,0 & 2,5 \\
50 & 41,3 & 7,4 & 2,5 \\
100 & 43,6 & 7,4 & 2,9
\end{tabular}

* valores representam a média das medidas realizadas nos dois anos de avaliação.

\section{Produção de frutos na primeira safra}

A primeira produção considerada expressiva de um pomar de macieiras de baixa a média densidade de plantio e de mudas 
não pré-formadas, como foi o caso do experimento, dá-se no terceiro ano após a implantação. A partir daí, a adubação de formação do pomar deixa de ser utilizada e passa-se a empregar a adubação de manutenção. Apesar de não se observar aumento no número de órgãos produtivos (Tabela 2), constatou-se aumento significativo no número de frutos por planta no primeiro ano de produção, que praticamente foi duplicado em relação à testemunha quando se aplicou a dose máxima de $100 \mathrm{~kg} \mathrm{ha}^{-1} \mathrm{de} \mathrm{N}$ (Figura 3). Isso indica que a nutrição das gemas foi, de alguma forma, afetada pela adubação nitrogenada nos anos iniciais. Possivelmente, pode ter ocorrido aumento do número de frutos por cacho floral em resposta à melhor nutrição das gemas que o geraram. Ressalta-se que, no raleio, foram mantidos, em média, mais do que um fruto/cacho floral. Neilsen et al. (1999) também observaram que o acréscimo de produção, em anos subseqüentes às adubações de crescimento, estava relacionado com o aumento do número de frutos por planta. No mesmo trabalho, observaram que a competição por água e nutrientes entre as plantas invasoras e a macieira, no período que antecedeu a fase produtiva, refletiu em decréscimos no rendimento de maçãs, nas duas primeiras safras, para a cultivar Gala. Resultados obtidos por Mattos et al.(2004) com a cultura da laranja mostraram que o aumento da dose de $\mathrm{N}$ aplicada aumentou o pegamento de frutos e, conseqüentemente, o número de frutos produzidos por unidade de volume de copa. Esse efeito está relacionado com o papel do $\mathrm{N}$ na regulação da taxa fotossintética e da síntese de carboidratos, do peso específico das folhas, da produção de biomassa total e da alocação de carbono em diferentes órgãos na planta, favorecendo a nutrição das gemas.

A massa média, o rendimento de frutos e o número de frutos por planta foram aumentados de forma linear em resposta às doses do fertilizante (Figura 3). Uma vez que a dose máxima de $\mathrm{N}$ utilizada no trabalho foi superior à recomendada (SBCS: Comissão de Química e Fertilidade do Solo, 2004), pode-se inferir que as recomendações atuais de adubação de crescimento para a macieira estão aquém das que deveriam ser usadas nas condições de solo e clima de São Joaquim, considerando-se a competição das plantas invasoras com a macieira que ocorreu no presente estudo. Essa inferência pode ser ressaltada pelo fato de que as doses aplicadas também foram insuficientes para que as folhas atingissem o nível normal de suficiência, conforme mostrado na Figura 1.

\section{Correlações entre as variáveis}

$\mathrm{Na}$ Tabela 3, estão apresentadas as correlações entre as variáveis medidas no terceiro ano após a primeira aplicação de N. Observa-se que a massa média dos frutos e o seu número por planta, ao contrário do que era de se esperar, apresentaram correlação positiva. Isto se deve provavelmente por tratar-se da primeira produção, onde o número de frutos por planta é relativamente baixo. $\mathrm{O}$ comprimento dos lançamentos foi aumentado em $4,8 \mathrm{~cm}$ com a dose máxima de $\mathrm{N}$ em relação à testemunha. Apesar de este aumento não ter sido significativo (Tabela 1), a correlação positiva com o número de frutos por planta e com o rendimento pode ser um indício de que esse pequeno aumento foi importante sobre a produtividade das plantas, mesmo porque a massa média dos frutos correlacionouse significativamente com a capacidade produtiva da copa (CP). A exemplo do que já foi observado em outros trabalhos (Stiles, 1994; Neilsen et al., 1999), houve alta relação entre o rendimento de frutos e o perímetro do tronco da macieira

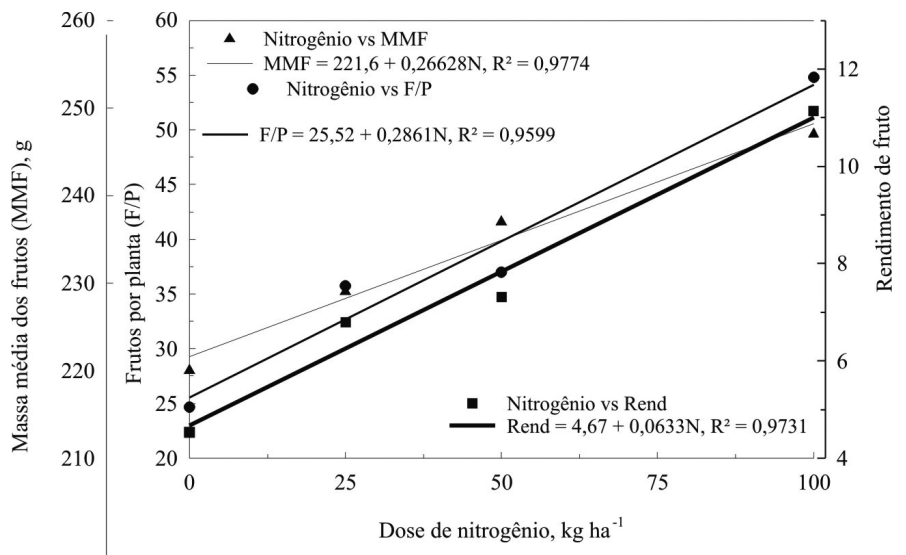

FIGURA 3 - Número de maçãs por planta (F/P), massa média dos frutos (MMF) e produtividade da macieira (Rend), cv. Catarina sobre porta-enxerto Marubakaido em função de doses de $\mathrm{N}$ no terceiro ano após a primeira fertilização, para as condições de São Joaquim-SC (2001). (As doses de $\mathrm{N}$ representam a soma das três aplicações anuais).

TABELA 3 - Correlações significativas $(\alpha=0,05)$ entre as variáveis avaliadas no terceiro ano após o início da aplicação dos tratamentos, para as condições de São Joaquim-SC (2000 e 2001).

\begin{tabular}{lccccc}
\hline \multicolumn{1}{c}{ Variáveis } & $\mathrm{Fr} /$ planta & Peso Méd. Fr. & Rendimento / ha & Lançamentos & Capacidade Prod. \\
\hline Peso Médio Frutos & 0,3827 & 1 & & & \\
Rendimento / ha & 0,9895 & 0,5028 & 1 & & \\
Lançamentos & 0,4220 & & 0,4146 & 1 & \\
Cap. Produtiva & & 0,5354 & & $0,2156^{*}$ & 1 \\
Perímetro tronco & 0,5998 & 0,4029 & 0,6106 & 0,5152 & 0,2681 \\
\hline
\end{tabular}

* correlação não-significativa

\section{CONCLUSÕES}

1-A capacidade produtiva da macieira não foi afetada pela adubação nitrogenada realizada na fase de formação e correlacionou-se positivamente com o tamanho dos frutos.

2-O teor de $\mathrm{N}$ nas folhas aumentou linearmente com as doses de $\mathrm{N}$ e somente atingiu o limite inferior da faixa normal de suficiência com doses superiores a $50 \mathrm{~kg} \mathrm{ha}^{-1} \mathrm{de} \mathrm{N}$.

3-O vigor vegetativo medido pelo perímetro do tronco foi aumentado pela adubação nitrogenada de crescimento.

4-O rendimento de frutos, o número de frutos por planta e a massa média dos frutos obtidos na primeira produção foram 
aumentados pelas adubações de crescimento realizadas em anos anteriores ao do início da produção.

\section{REFERÊNCIAS}

BASSO, C.; SUZUKI, A. Resposta da macieira cv. Golden Delicious à adubação nitrogenada. Revista Brasileira de Ciência do solo, Campinas, v.16, n.2, p.223-227, 1992.

COMISSÃO DE FERTILIDADE DO SOLO - RS/SC. Recomendações de adubação e de calagem para os estados do Rio Grande do Sul e de Santa Catarina. 3.ed. Passo Fundo: SBCS-Núcleo Regional Sul, 1995. 224p.

COMISSÃO DE QUÍMICA E FERTILIDADE DO SOLO. Manual de adubação e calagem para os estados do Rio Grande do Sul e de Santa Catarina. 10.ed. Porto Alegre: SBCS-NRS, 2004. $400 \mathrm{p}$.

DAUGAARD, H.; GRAUSLUND J. Fruit colour and correlations with orchard factors and post-harvest characteristics in apple cv. Mutsu. Journal of Horticultural Science \& Biotechnology, Valência, v.74, n3, p.283-287, 2000.

EBERT, A.; RAASCH, Z. S. Sistemas de plantio para macieiras. In: EMPASC. Manual da cultura da macieira. FlorianópolisSC, 1987. p.236-265.

ERNANI, P.R.; DIAS, J.; VANZ, L. Application of nitrogen to the soil after fruit harvest has not increased apple yield. Revista Brasileira de fruticultura, Cruz das Almas, v. 9, n.1, p.33-37, 1997.

IUCHI, V. L.; NAVA, G.; IUCHI, T. Distúrbios fisiológicos e desequilíbrios nutricionais em macieira. Florianópolis: Epagri/Jica, 2001. 74p.

MANRU, G.; HUAIRUI, S.; HONGWEI, Z. A study on nitrogen nutrition of apple trees. In: Diagnosis of nutritional status of deciduous fruit orchards. Acta Horticulturae, Wageningen, v.274, p.179-185, 1990.

MATTOS, J. D.; QUAGGIO, J. A.; CANTARELlA, H.; CARVALHO, S. A. Superfícies de resposta do tangor 'Murcott' à fertilização com N, P e K. Revista Brassileira de Fruticultura, Jaboticabal, v.26, n.1, p.164-167, 2004.

NAVA G.; NUERNBERG N.J., KATSURAYAMA, J.M.; WATANABE M. Resposta da macieira cv Fuji à adubação nitrogenada e potássica. In: REUNIÃO SUL BRASILEIRA DE CIÊNCIA DO SOLO, 3., 2000. Pelotas. Rumos da ciência do solo para o desenvolvimento do Rio Grande do Sul e Santa Catarina: resumos... Pelotas: EMBRAPA Clima Temperado e Núcleo Regional Sul da Sociedade brasileira de Ciência do Solo, 2000. CD-ROM

NEILSEN,G. H.; HOGUE, E. J.; MEHERIUK, M. Nitrogen fertilization and orchard-floor vegetation management affect growth, nutrition and fruit quality of gala apple. Canadian Journal of Plant Science, Ottawa, v.79, p.379-385, 1999.

NEILSEN, D.; MILLARD, P.; NEILSEN, G. H.; HOGUE, E. J. Nitrogen uptake, efficiency of use, and partioning for growth in young apple trees. Journal of the American Society for Horticultural Science, Alexandria, v.126, n.1, p. 144-150, 2001. PROENÇA, M. M.; ERNANI, P. R.; DIAS, J. Rendimento e qualidade de frutos de macieira influenciados pela aplicação de N mineral em pomar com porta-enxerto anão. In: REUNIÃO SUL BRASILEIRA DE CIÊNCIA DO SOLO, 4., 2002. Porto Alegre. Solos e qualidade ambiental: resumos... Porto Alegre: Universidade Federal do Rio Grande do Sul e Núcleo Regional Sul da Sociedade Brasileira de Ciência do Solo, 2002. CDROM

SAS INSTITUTE. The SAS System - Release 6.12. Cary, NC. 1996. STILES, W.C. Nitrogen management in the orchard. In: PETERSON, A.B.; STEVENS, R.G. (Eds). Tree fruit nutrition. Yakima, WA: Good Fruit Grower, 1994 . p.41-50.

SUZUKI, A.; BASSO, C. Fertilidade do solo e nutrição da macieira. In: EPAGRI. Manual da cultura da macieira. Florianópolis, 2002. p.341-381. 